\title{
Regulating Subcellular Metal Homeostasis: The Key to Crop Improvement
}

\author{
Khurram Bashir $^{1 *}$, Sultana Rasheed ${ }^{1,2}$, Takanori Kobayashi ${ }^{3}$, Motoaki Seki $^{1,2,4}$ and \\ Naoko K. Nishizawa ${ }^{3,5 *}$
}

1 Plant Genomics Network Research Team, Center for Sustainable Resource Science, RIKEN, Yokohama Campus, Yokohama, Japan, ${ }^{2}$ Kihara Institute for Biological Research, Yokohama City University, Yokohama, Japan, ${ }^{3}$ Research Institute for Bioresources and Biotechnology, Ishikawa Prefectural University, Nonoichi, Japan, ${ }^{4}$ Core Research for Evolutional Science and Technology - Japan Science and Technology Agency, Kawaguchi, Japan, ${ }^{5}$ Graduate School of Agricultural and Life Sciences, The University of Tokyo, Tokyo, Japan

\section{OPEN ACCESS}

Edited by:

Swapan K. Datta,

University of Calcutta, India

Reviewed by:

Stephan Clemens,

University of Bayreuth, Germany Marc Hanikenne,

University of Liège, Belgium

*Correspondence:

Khurram Bashir

khurram.bashir@riken.jp

Naoko K. Nishizawa

annaoko@mail.ecc.u-tokyo.ac.jp

Specialty section:

This article was submitted to Plant Nutrition,

a section of the journal

Frontiers in Plant Science

Received: 12 April 2016

Accepted: 25 July 2016

Published: 05 August 2016

Citation:

Bashir K, Rasheed S, Kobayashi T,

Seki M and Nishizawa NK (2016)

Regulating Subcellular Metal Homeostasis: The Key to Crop Improvement.

Front. Plant Sci. 7:1192. doi: 10.3389/fpls.2016.01192
Iron $(\mathrm{Fe})$, zinc $(\mathrm{Zn})$, manganese $(\mathrm{Mn})$, and copper $(\mathrm{Cu})$ are essential micronutrient mineral elements for living organisms, as they regulate essential cellular processes, such as chlorophyll synthesis and photosynthesis ( $\mathrm{Fe}, \mathrm{Cu}$, and $\mathrm{Mn}$ ), respiration (Fe and $\mathrm{Cu}$ ), and transcription $(\mathrm{Zn})$. The storage and distribution of these minerals in various cellular organelles is strictly regulated to ensure optimal metabolic rates. Alteration of the balance in uptake, distribution, and/or storage of these minerals severely impairs cellular metabolism and significantly affects plant growth and development. Thus, any change in the metal profile of a cellular compartment significantly affects metabolism. Different subcellular compartments are suggested to be linked through complex retrograde signaling networks to regulate cellular metal homeostasis. Various genes regulating cellular and subcellular metal distribution have been identified and characterized. Understanding the role of these transporters is extremely important to elaborate the signaling between various subcellular compartments. Moreover, modulation of the proteins involved in cellular metal homeostasis may help in the regulation of metabolism, adaptability to a diverse range of environmental conditions, and biofortification. Here, we review progress in the understanding of different subcellular metal transport components in plants and discuss the prospects of regulating cellular metabolism and strategies to develop biofortified crop plants.

Keywords: biofortification, chloroplast, iron, manganese, metabolome, metal transport, mitochondria, zinc

\section{INTRODUCTION}

Metals, such as iron $(\mathrm{Fe})$, zinc $(\mathrm{Zn})$, copper $(\mathrm{Cu})$, and manganese $(\mathrm{Mn})$, are essential for all higher organisms (Marschner, 1995). Fe readily changes its oxidative state, which allows participation in cellular functions, and its regulation is extremely important to avoid cellular toxicity. Fe participates in cellular respiration, synthesis, and stabilization of chlorophyll, photosynthetic electron transport, and various other metabolic functions (Grotz and Guerinot, 2006). The most obvious symptom of Fe-deficiency in plants is chlorosis due to a decrease in chlorophyll content, which significantly affects plant growth, development and product quality. $\mathrm{Cu}$ is also a 
cofactor for various enzymes involved in respiration and photosynthesis and is toxic at higher concentrations. In plants, Zn does not readily change its oxidative state and does not take part in oxidoreduction reactions (Marschner, 1995). $\mathrm{Zn}$ is integral to enzymes involved in protein, nucleic acid, carbohydrate, and lipid metabolism (Ishimaru et al., 2011; Suzuki et al., 2012). $\mathrm{Zn}$ is also critical for proteins containing DNA-binding $\mathrm{Zn}$ finger motifs, RING fingers, LIM domains, proteins associated with DNA and RNA synthesis, such as transcription factors, RNA polymerases, and reverse transcriptase (Broadley et al., 2007; Bashir et al., 2012). Mn is a cofactor or activator of enzymes, such as oxalate oxidase, Mn superoxide dismutase, RNA polymerase, malic enzyme, isocitrate dehydrogenase, and phosphoenolpyruvate carboxykinase, and it is required for photosynthetic oxygen evolution in chloroplasts (Marschner, 1995; Socha and Guerinot, 2014).

Various cellular organelles, such as chloroplasts and mitochondria, depend on $\mathrm{Fe}, \mathrm{Cu}, \mathrm{Mn}$, and $\mathrm{Zn}$ for their activities, and deficiencies and toxicity of these minerals significantly disturb chloroplast and mitochondrial functioning, which ultimately hinders plant growth and development (Bashir et al., 2011a,b; Duy et al., 2011; Vigani et al., 2013b). Thus, regulating the uptake and cellular distribution of these minerals is extremely important for optimal cellular functioning and could lead to breeding crop plants with better adaptability to changing environments and may contribute to providing healthy food with improved mineral contents. Regulation of Fe uptake from the rhizosphere has been reviewed extensively (Kobayashi and Nishizawa, 2012). Genes involved in Fe acquisition in plants are induced under Fe-deficient conditions, and various genes putatively acting as Fe sensors have been discussed (Kobayashi and Nishizawa, 2014). Uptake of $\mathrm{Zn}, \mathrm{Cu}$, and $\mathrm{Mn}$ is also reasonably well understood and has been reviewed (Palmer and Guerinot, 2009; Yruela, 2009; Bashir et al., 2012; Jain et al., 2014; Socha and Guerinot, 2014). Here, we review the proteins controlling mineral distribution in various cellular organelles and discuss the prospects of regulating these proteins for optimized metabolism and biofortification purposes.

\section{CELLULAR METAL HOMEOSTASIS}

Plants absorb metals from the soil for transport to the cytoplasm and specific subcellular compartments, such as the nucleus, mitochondria, and chloroplasts, where they perform specific functions, whereas vacuoles serve as a reservoir to regulate cell metallic balance. Cellular metal transport is governed by a diverse set of membrane proteins. As free metals are toxic, plants utilize various chelating agents, such as nicotianamine (NA), deoxymugineic acid (DMA), citrate, ascorbate, and phenolics, to solubilize these metals and protect cells from oxidative damage (Jeong and Guerinot, 2009; Palmer and Guerinot, 2009; Bashir et al., 2013a; Grillet et al., 2014). Transporters belonging to various families actively participate in the regulation of cellular metal homeostasis (Vigani et al., 2013a; Boutigny et al., 2014; Jain et al., 2014; Socha and Guerinot, 2014; Finazzi et al., 2015; López-Millán et al., 2016).
Various members of the Yellow stripe-like (YSL) family (named for the phenotype of mutants from which the maize Yellow Stripe 1 was cloned), zinc-regulated transporter/iron-regulated transporter (ZRT/IRT)-related protein (ZIP) family, natural resistance associated macrophage protein (NRAMP) family, cation diffusion facilitator (CDF) family, major facilitator super family (MFS), $\mathrm{P}_{1 \mathrm{~B}}$-type heavy metal ATPase (HMA) family, the vacuolar iron transporter (VIT) family, and the cation exchange (CAX) family play significant roles in cellular metal homeostasis (Figure 1). Understanding the function and regulation of these transporters is extremely important to characterize the factors governing micronutrient uptake and distribution in plants.

\section{CHLOROPLAST METAL HOMEOSTASIS}

Metals play an extremely important role in chlorophyll synthesis, stabilization and photosynthesis; thus, regulating metal homeostasis in chloroplasts is extremely important (Finazzi et al., 2015; López-Millán et al., 2016). In plants, the Fe storage protein ferritin localizes mainly to chloroplasts; thus, chloroplasts serve as a storage hub for $\mathrm{Fe}$, particularly during early plant growth (Briat et al., 2010). The roles of $\mathrm{Fe}, \mathrm{Mn}$, and $\mathrm{Cu}$ in photosynthesis and metabolism have been discussed extensively (Nouet et al., 2011; Vigani et al., 2013a; López-Millán et al., 2016), so we will not discuss these details here. The Arabidopsis ferric chelate reductase oxidase 7 (FRO7), which reduces ferric ion to $\mathrm{Fe}^{2+}$, localizes to chloroplasts and plays a significant role in Fe homeostasis (Jeong et al., 2008; Jain et al., 2014). Permease in chloroplasts 1 (PIC1) is important for Fe transport to chloroplasts (Duy et al., 2011), and the role of Arabidopsis mitoferrin-like 1 (AtMfl1) in Fe transport to chloroplasts has also been discussed, although there is no direct evidence that AtMfl1 transports Fe (Tarantino et al., 2011). An increase or decrease in PIC1 expression significantly alters chloroplast development and plant growth through reactions triggered by Fe toxicity or Fe-deficiency. These results indicate that reciprocal signaling between chloroplasts and nuclear genes may exist (Duy et al., 2011; López-Millán et al., 2016). Contrasting reports on the localization and function of AtYSL4 and AtYSL6 have been published (Conte et al., 2013; Divol et al., 2013). According to Divol et al. (2013), Arabidopsis YSL4 and YSL6 localize to chloroplasts and play a major role in releasing chloroplastic Fe-NA, with a particularly important role during embryogenesis and senescence.

Importation of $\mathrm{Cu}$ into chloroplasts is mediated mainly by a chloroplast-envelope $\mathrm{P}_{\mathrm{IB}}$-type ATPase, called HMA6 (also known as PAA1), which transports $\mathrm{Cu}^{+}$and is required for photosynthesis (Catty et al., 2011; Boutigny et al., 2014). Copper may also be imported by HMA1, which transports $\mathrm{Cu}^{2+}$ (Boutigny et al., 2014). HMA1 and HMA6 behave as distinct pathways for importing $\mathrm{Cu}$ (Boutigny et al., 2014). The HMA1 and HMA6 double mutants do not exhibit a lethal phenotype, suggesting that $\mathrm{Cu}$ could enter the chloroplast through another, yet unknown, transporter (Catty et al., 2011; Boutigny et al., 2014). The role of HMA8 (PAA2) in Cu transport to chloroplast thylakoid membrane has also been documented (Aguirre and Pilon, 2016). 


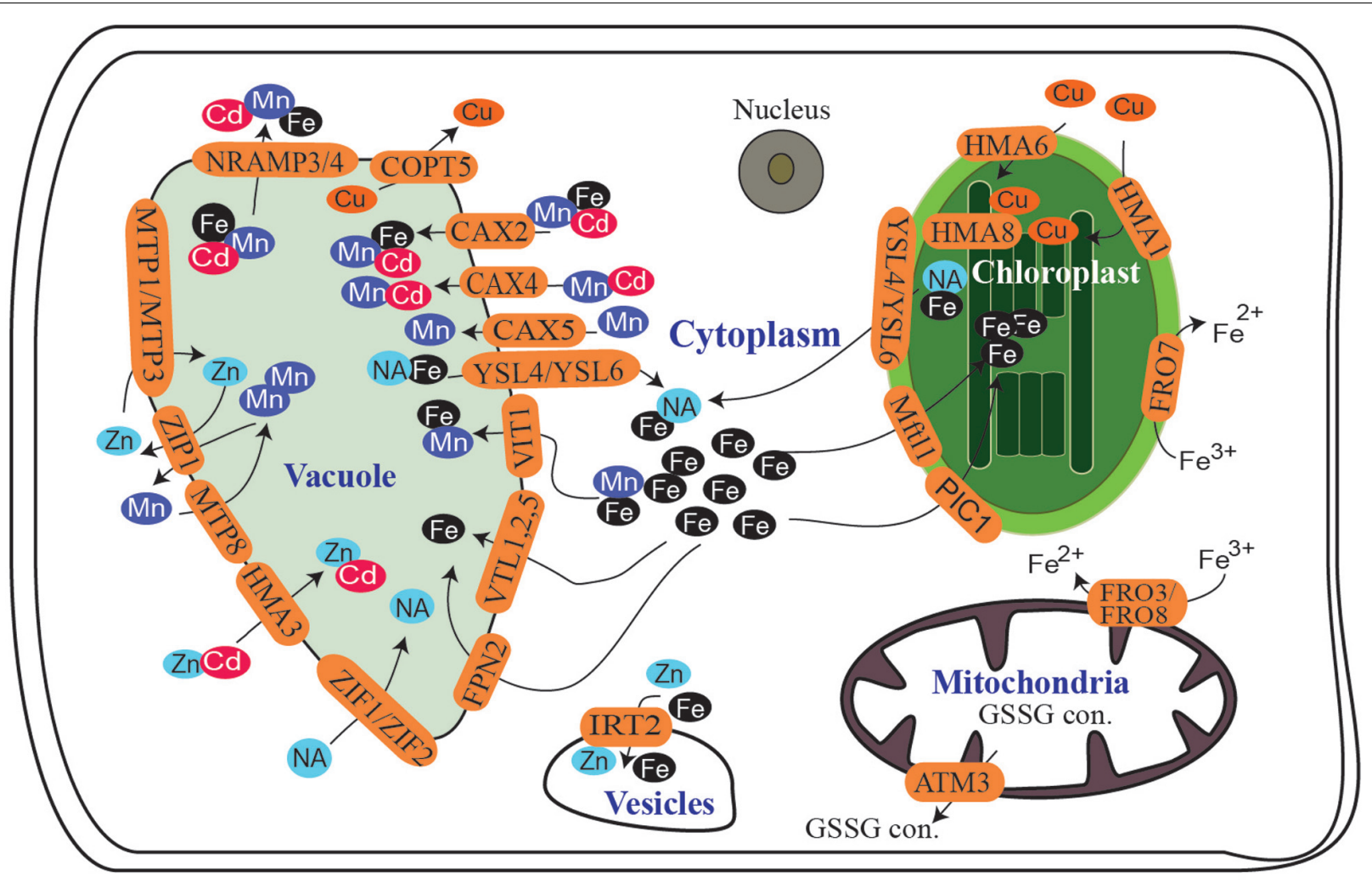

FIGURE 1 | Summary of subcellular metal transport in Arabidopsis. Proteins participating in Fe, Mn, Cu, or Zn transport into or out of different cellular organelles are shown. NA, nicotianamine; GSSG con., glutathione conjugates.

Despite the essential role of $\mathrm{Mn}$ in photosynthesis, Mn transporters localizing to the chloroplast membrane have not been reported. Similarly, although HMA1 is suggested to be involved in the control of $\mathrm{Zn}$ homeostasis in chloroplasts (Williams and Mills, 2005; Kim et al., 2009), its role in $\mathrm{Zn}$ transport is controversial (Boutigny et al., 2014), and the protein(s) transporting $\mathrm{Zn}$ to and from chloroplasts have not been clearly documented. Characterization of chloroplast transporters would provide a way to regulate metal homeostasis in chloroplasts and ultimately improve crops. Regulating metal transport to and from the chloroplast could be utilized to improve photosynthesis, improve cellular metabolism, and develop crop plants with a better ability to grow under changing environmental conditions.

\section{THE VACUOLE IS A MAJOR RESERVOIR FOR METAL HOMEOSTASIS}

The vacuole serves as a major reservoir to regulate cellular metal homeostasis. To avoid metal toxicity, excess metals are deposited in vacuoles for withdrawal when required. In plants, several efflux and influx transporters localized to the vacuolar membrane have been reported. In Arabidopsis, FPN2 (also known as IREG2) deposits cytoplasmic Fe in vacuoles, whereas vacuolar Fe transporter 1 (VIT1) deposits Fe and Mn in vacuoles
(Kim et al., 2006; Schaaf et al., 2006; Morrissey et al., 2009). Changes in VIT1 expression significantly alters Fe localization in Arabidopsis seeds (Kim et al., 2006). Arabidopsis VIT1-like (VTL) proteins have also been characterized (Gollhofer et al., 2014). Arabidopsis VTL1, VTL2, and VTL5 are functional Fe transporters that play a role in transferring cytoplasmic $\mathrm{Fe}$ into vacuoles and contribute to Fe homeostasis regulation in planta (Gollhofer et al., 2014). It is unclear why Arabidopsis requires so many vacuolar Fe transporters and whether VTL proteins also transport other metals. In rice, VIT1 and VIT2 are functional vacuolar transporters that contribute to $\mathrm{Fe}, \mathrm{Mn}$, and Zn homeostasis. Disrupting VIT1 or VIT2 function leads to increased metal accumulation in rice seeds (Zhang et al., 2012). Disrupting VIT also alters petal colors in flowers, due to changes in the accumulation of pigments that complex with metals (Momonoi et al., 2009; Yoshida and Negishi, 2013).

Natural resistance associated macrophage proteins are integral membrane proteins involved in transport of $\mathrm{Mn}, \mathrm{Fe}$, and $\mathrm{Cd}$. The mobilization of Fe (as well as $\mathrm{Mn}$ and $\mathrm{Cd}$ ) from vacuoles in Arabidopsis is mediated by NRAMP3 and NRAMP4. Arabidopsis NRAMP3 and NRAMP4 release Fe from vacuoles under limited $\mathrm{Fe}$ conditions or when demand increases (Lanquar et al., 2005; Mary et al., 2015; Pottier et al., 2015). AtNRAMP3 and AtNRAMP4 function oppositely to AtVIT1. One study revealed that preventing $\mathrm{Fe}$ storage in endodermal vacuoles through 
AtVIT1 knockout rescues the Fe mobilization defect in the nramp3nramp4 double mutant under optimal conditions (Mary et al., 2015).

The role of Arabidopsis YSL4 and YSL6 in remobilizing vacuolar Fe has also been discussed (Conte et al., 2013). In contrast to Conte et al. (2013), Divol et al. (2013) reported that AtYSL4 and AtYSL6 localize to vacuolar and internal membranes resembling endoplasmic reticulum (ER) and were suggested to play a role in heavy metal stress responses. AtYSL4 and AtYSL6 knockout mutants grow better under excess $\mathrm{Mn}$ and exhibit improved growth of roots exposed to excess $\mathrm{Mn}$ and excess nickel (Ni), whereas overexpression of YSL6 results in decreased root growth under excess $\mathrm{Mn}$ or $\mathrm{Ni}$. In this context, it is reasonable to believe that AtYSL4 and AtYSL6 may help provide Mn and Ni-NA complexes to proteins located in internal cellular compartments (Conte et al., 2013).

Metal tolerant protein 1 (MTP1) and MTP3 in Arabidopsis are critical for sequestering excess $\mathrm{Zn}$ into the vacuole (DesbrossesFonrouge et al., 2005; Arrivault et al., 2006). Zn deposition in the vacuole is also mediated by HMA3, which sequesters Cd (Morel et al., 2009). Arabidopsis ZIP1 is a vacuolar $\mathrm{Zn}$ and Mn transporter and may play a role in remobilizing $\mathrm{Mn}$ and/or $\mathrm{Zn}$ from the vacuole to the cytoplasm in roots (Milner et al., 2013). Efflux of NA 1 (ENA1) is a member of the MFS family that transports NA to the vacuole in rice (Nozoye et al., 2011). ENA1 homologs, Zn-induced facilitator 1 (ZIF1) and ZIF2, have been characterized in Arabidopsis (Haydon and Cobbett, 2007; Haydon et al., 2012; Remy et al., 2014). Arabidopsis ZIF1 and ZIF2 localize to the vacuole and may play a role in tolerance to excess $\mathrm{Zn}$ (Haydon and Cobbett, 2007; Haydon et al., 2012; Remy et al., 2014). The ZIF1 could also play important role in Fe transport under Fe deficient conditions (Haydon et al., 2012). The role of ZIFL2 to mediate potassium and cesium influx in Arabidopsis has also been reported recently (Remy et al., 2015).

Arabidopsis CAX2 transports $\mathrm{Fe}, \mathrm{Cd}$, and $\mathrm{Mn}$; CAX4 transports $\mathrm{Mn}$ and $\mathrm{Cd}$, and CAX5 transports $\mathrm{Mn}$ to the vacuole (Socha and Guerinot, 2014). The role of VIT1 in sequestering $\mathrm{Mn}$ to the vacuole has also been discussed. Arabidopsis MTP8 belongs to the CDF family of transporters and was identified by screening T-DNA mutants on high $\mathrm{pH}$ medium with decreased Fe availability (Eroglu et al., 2016). MTP8 is a vacuolar Mn transporter, which sequesters excess $\mathrm{Mn}$ to the vacuole, and plays a major role protecting plants from Mn toxicity. MTP8 expression increases under low Fe conditions as well as under high Mn conditions (Eroglu et al., 2016). mtp8 mutants are more sensitive to Fe-deficiency in the presence of Mn due to the decrease in ferric chelate reductase activity (Eroglu et al., 2016). Alternately this increased sensitivity could also result from an inability to store the Mn taken up by ZIP family Fe regulated transporter 1 (IRT1). IRT1, is involved in $\mathrm{Cd}, \mathrm{Fe}, \mathrm{Mn}$, and $\mathrm{Zn}$ transport into the cell. Unlike other plants, rice accumulates more $\mathrm{Mn}$ in shoots compared with roots (Ishimaru et al., 2012), and rice MTP8.1 seems to be the major gene sequestering excess Mn into the vacuole (Chen et al., 2013). OsVIT1 and OsVIT2 may also contribute to sequestration of $\mathrm{Mn}$ into the vacuole (Zhang et al., 2012). Vacuolar Mn remobilization in Arabidopsis is controlled by NRAMP3, NRAMP4, and ZIP1. The Arabidopsis
$\mathrm{Cu}$ transporter (COPT/Ctr) family members COPT1, COPT2, COPT3, and COPT5 have been implicated in $\mathrm{Cu}$ transport (Grotz and Guerinot, 2006). Among these, COPT5 pumps vacuolar $\mathrm{Cu}$ into the cytoplasm and plays an integral role in photosynthetic electron transport in chloroplasts (Garcia-Molina et al., 2011; Klaumann et al., 2011).

Mineral transporters also interact with toxic metals as described above. For example, HMAs are members of a diverse family of transporters localized in the plasma membrane as well as subcellular compartments, and they are involved in translocation or detoxification of $\mathrm{Cu}, \mathrm{Zn}$, and $\mathrm{Cd}$. Arabidopsis HMA3 sequesters $\mathrm{Cd}$ and $\mathrm{Zn}$, (may also be involved in Co, and $\mathrm{Pb}$ sequestration) to the vacuole, whereas OsHMA3 seems very specific to Cd sequestration (Morel et al., 2009; Ueno et al., 2010; Miyadate et al., 2011). In Arabidopsis, CAX2, CAX4. NRAMP3 and NRAMP4 also transport Cd. The expression of vacuolar transporters may or may not be regulated by the cytoplasmic status of the plants; e.g., ZIF1 (Haydon and Cobbett, 2007), OsVIT2 (Zhang et al., 2012), CAX4 (Mei et al., 2009), which regulate metal sequestration into vacuoles, are upregulated under excess metal conditions. MTP3 expression is positively regulated by Fe deficiency and by excess $\mathrm{Zn}$ and Co (Arrivault et al., 2006). On the other hand, Arabidopsis VIT1 expression (Kim et al., 2006; Zhang et al., 2012), MTP1 (Kobae et al., 2004; Kawachi et al., 2009) and COPT5 (Garcia-Molina et al., 2011; Klaumann et al., 2011) remain unaffected by metal status (Peng and Gong, 2014). It is important to mention that basic helixloop-helix protein FER-like Fe deficiency-Induced Transcription factor (FIT) regulates the expression of various genes (such as IREG2, MTP3, MTP8) to cope with metal excess resulting from increased expression of IRT1 under Fe deficiency (Schmidt and Buckhout, 2011).

\section{MITOCHONDRIAL METAL HOMEOSTASIS}

Mitochondria have evolved through endosymbiosis and retain numerous functions, such as synthesis of heme and lipoic acid cofactors, as well as assembly of bacterial Fe-S cluster (ISC) machinery (Lill et al., 2015). The ISC components are essential for other cellular compartments, as assembly of cytosolic and nuclear $\mathrm{Fe} / \mathrm{S}$ proteins depends on the generation of mitochondrial S-containing compounds (Lill et al., 2015). Arabidopsis ATM3 (also known as STA1) exports glutathione trisulfide complexes from mitochondria to the cytoplasm (Schaedler et al., 2014), and defects in transport significantly affect Fe-S cluster assembly and molybdenum cofactor assembly in the cytosol (Kushnir et al., 2001; Bernard et al., 2009; Teschner et al., 2010), leaving plants chlorotic. In addition to its role in $\mathrm{Fe}-\mathrm{S}$ cluster assembly, glutathione plays an important role in the plant's response to metal availability (Bashir et al., 2007; Shanmugam et al., 2015). Glutathione plays an essential role in $\mathrm{Fe}$ deficiency tolerance and NO-mediated Fe-deficiency signaling in Arabidopsis (Shanmugam et al., 2015).

Based on the vital functions of mitochondria in plants, it seems reasonable to conclude that mitochondria and/or other 
cellular organelles may regulate retrograde signaling pathways to control Fe deficiency-induced responses; however, the nature of the pathway is not clear (Vigani et al., 2013a,b). Plant mitochondria serve as a central hub of redox regulation and energy conversion by linking metabolic pathways from different subcellular compartments. These properties make mitochondria an ideal sensor to reflect cellular energetic and metabolic statuses (Sweetlove et al., 2007; Millar et al., 2011). As mitochondria serve as the powerhouse of the cell, changes in cellular energy status can reconfigure mitochondrial activities. As a result, changes in mitochondrial status could affect functions in other cellular compartments, such as changes in photosynthetic activity or nuclear gene expression (Schwarzländer et al., 2012; Vigani et al., 2013b).

Transport of metals into plant mitochondria is poorly understood, as only a few proteins involved in metal transport to and from mitochondria have been characterized. Arabidopsis FRO3 and FRO8 are mitochondrial proteins that putatively participate in ferric reduction in the mitochondrial membrane and contribute to mitochondrial Fe transport (Jain et al., 2014). The protein that transports cytoplasmic $\mathrm{Fe}$ into mitochondria has been characterized in rice. The rice mitochondrial $\mathrm{Fe}$ transporter (MIT) is essential for growth and development, as the rice MIT knockout mutant (mit-1) is lethal, and the MIT knockdown mutant (mit-2) exhibits reduced chlorophyll content and significantly affected plant growth. mit-2 plants accumulate less $\mathrm{Fe}$ in mitochondria and significantly higher $\mathrm{Fe}$ in leaves and show symptoms of Fe deficiency, i.e., reduced chlorophyll content and upregulation of genes normally induced by $\mathrm{Fe}$ deficiency (Bashir et al., 2011a,b). Partial loss of MIT function in rice results in altered respiration and decreased total and mitochondrial aconitase activities, which possibly affect synthesis of the $\mathrm{Fe}-\mathrm{S}$ cluster at the mitochondrial and cytosolic levels (Bashir et al., 2011b; Vigani et al., 2016). The yeast mitochondrial Fe transporter MRS3 also contributes to $\mathrm{Cu}$ transport (Vest et al., 2016). It is unclear if MIT contributes to transport of other divalent cations, particularly $\mathrm{Cu}$. $\mathrm{Mn}$ accumulation in leaves as well as $\mathrm{Mn}$ and $\mathrm{Cu}$ accumulation in isolated mitochondria of mit-2 plants is significantly altered (Bashir et al., 2011b). These changes could be due to either upregulation of other metal transporters, such as OsVIT2, or a direct effect of reduced MIT activity. The transporters involved in mitochondrial Mn, $\mathrm{Cu}$, or $\mathrm{Zn}$ homeostasis have not been reported in plants. Two homologs of MIT have been identified in Arabidopsis (Jain, 2014).

Besides these transporter proteins, mitochondrial ferritin and frataxin also play a role in mitochondrial metal homeostasis. Fer4 in Arabidopsis localizes to mitochondria (Murgia and Vigani, 2015). Defects in Arabidopsis frataxin result in an embryonic lethal phenotype; thus, a frataxin knockout mutant can only be maintained as a heterozygote (Murgia and Vigani, 2015). Mitochondrial respiration is not affected in ferritin- or frataxinimpaired Arabidopsis; however, these disruptions significantly alter accumulation of several minerals in Arabidopsis leaves. The mitochondrial iron regulated (MIR) protein in rice plays an undefined role in metal homeostasis. MIR expression is significantly upregulated in response to Fe deficiency, and the expression of metal homeostasis-related genes and accumulation of Fe changes significantly in MIR knockout mutants (Ishimaru et al., 2009). The MIR seems very specific to rice, as its homologs have not been identified in other crop species (Ishimaru et al., 2009).

\section{VESICLES AND THE GOLGI COMPLEX PLAY A SIGNIFICANT ROLE IN CELLULAR METAL HOMEOSTASIS}

Metal distribution in the nucleus, Golgi complex, and vesicles is important for cellular function (Roschzttardtz et al., 2011; Seo et al., 2012), and various proteins localized to these compartments have been described. The nucleus contains a significant amount of Fe (Roschzttardtz et al., 2011); however, it is not clear if transporters contributing to $\mathrm{Fe}$ transport (and other metals) into the nucleus exist in plants. The pore size of nucleus is large enough to allow metal movement, however, the excess metals may be extremely toxic for the nucleus thus there is possibility that nucleus may be regulating the metal movement. Arabidopsis IRT2 localizes to vesicles within root epidermal cells (Conte and Walker, 2011). AtIRT2 transports $\mathrm{Fe}^{2+}$ and is regulated by Fe deficiency. Arabidopsis MTP11 plays a role in Mn transport and tolerance by sequestering $\mathrm{Mn}$ into internal organelles (Delhaize et al., 2007).

Rice YSL6 knockout mutants are specifically sensitive to high Mn concentrations, and OsYSL6 is suggested to be involved in detoxifying excess $\mathrm{Mn}$ in rice; however, its cellular localization is not clear (Sasaki et al., 2011). Barley YSL5 localizes to vesicle membranes and is suggested to play a role in transport of $\mathrm{Fe}$ and/or mugineic acids (Zheng et al., 2011). HvYSL5 expression is specifically regulated by $\mathrm{Fe}$ and not by $\mathrm{Cu}, \mathrm{Zn}$, or $\mathrm{Mn}$. It is also possible that OsYSL5 and/or OsYSL6 function in transporting the metal-NA or metal-DMA complex into internal compartments. HvYSL5, OsYSL5, and OsYSL6 formed a distinct clade with AtYSL4 and AtYSL6 in a phylogenetic analysis; thus, these transporters may also play a role in subcellular metal homeostasis in barley (HvYSL5) and rice (OsYSL5 and OsYSL6; Aoyama et al., 2009; Zheng et al., 2011). Interestingly, proteins encoded by NAS genes localize to vesicles in rice but not in Arabidopsis (Nozoye et al., 2014a,b); thus, it would be very interesting to determine if the roles of OsYSL5 and OsYSL6 are different from those of Arabidopsis YSL4 and YSL6. Metal transporters have also been identified in the endomembrane system, transporting metals to the ER and/or Golgi. Golgi-localized barley MTP8.1 and MTP8.2 are Mn efflux transporters (Pedas et al., 2014). MTP8.1 expression increases under Mn deficiency and toxicity in barley roots, whereas expression of MTP8.2 decreases. MTP8.1 and MTP8.2 expression in leaves decreases in response to excess $\mathrm{Mn}$. These proteins are suggested to play a significant role in Mn loading into the Golgi apparatus and in delivering Mn via secretory vesicles (Pedas et al., 2014). Arabidopsis HMA7 (also called RAN1) transports $\mathrm{Cu}$ for synthesis of ethylene receptors (Binder et al., 2010), and the IAR1 may be involved in $\mathrm{Zn}$ transport to ER (Lasswell et al., 2000). 


\section{REGULATING METABOLISM BY MANIPULATING METAL HOMEOSTASIS}

Metals compete in that increased availability of one metal significantly alters transport of other metals (Bashir et al., 2014; Vigani et al., 2016). The cellular response to metal accumulation is very complex and employs different signaling mechanisms. These responses can be triggered by transporters moving more than one metal or due to increased competition among metals. Changes in the accumulation of metals in different subcellular compartments significantly affect the genes involved in metal transport and are also suggested to affect metabolism (Vigani et al., 2013a, 2016; Bashir et al., 2014). The metal requirement of chloroplasts is particularly high. Photosynthesis is the major activity of chloroplasts and a site of primary and secondary metabolism; thus, it is not surprising that changes in the accumulation of $\mathrm{Fe}$ or other metals alter chloroplast activities and, in turn, nuclear gene expression and cellular metabolism. Mutants and PIC1-overexpressing plants significantly alter chloroplast development and plant growth through Fe toxicity or Fe-deficiency responses (Duy et al., 2011; López-Millán et al., 2016), indicating the possibility of reciprocal signaling between chloroplasts and nuclear genes (Duy et al., 2011; López-Millán et al., 2016). Thus regulating the expression of PIC1 through a stress inducible promoter could be an important strategy to regulate the optimal cellular metabolism and ultimately plant growth and development.

Transcriptomic and metabolomic profiling of mit-2 reveals that retrograde signaling between the nucleus and mitochondria significantly modulates cellular gene expression and metabolism (Vigani et al., 2016). Interestingly, these changes are differentially regulated in roots and shoots, highlighting the role of mitochondria in response to the different energy needs of these tissues. In general, more significant changes are observed in the shoot transcriptome and metabolome of mit-2 plants compared with those in root tissue. For example, oligosaccharides in the raffinose family along with pyruvic acid, fumaric acid, and ornithine specifically accumulate in shoot tissue (Vigani et al., 2016). The rice OsOPT7 mutant (opt7-1) accumulates twofold more $\mathrm{Fe}$ in shoots compared with that in wild-type plants (Bashir et al., 2011b, 2015). This phenotype significantly resembles mit2 phenotype, however, the transcriptomic changes in opt7-1 are significantly different from those in mit-2, mainly because Fe content and functionality are significantly altered in mit2 mitochondria. However, some changes may be attributed to differential storage of Fe. Additional $\mathrm{Fe}$ is probably stored in vacuoles in the mit-2 mutant versus in chloroplasts in the osopt7-1 mutant (Bashir et al., 2011b, 2015; Vigani et al., 2016). Moderately increased availability of metals seems to trigger metabolism (Bashir et al., 2014), thus carefully regulating metal transporters to deliver metal supply to different subcellular compartments may be a way to improve metabolism and ultimately crop yield and product quality.

It has already been reported that changes in vacuolar and mitochondrial $\mathrm{Fe}$ transporters affect metal localization in Arabidopsis and rice seeds (Kim et al., 2006; Zhang et al., 2012; Bashir et al., 2013b; Mary et al., 2015). Thus, carefully regulating the expression of subcellular metal transporters may help to biofortify food crops with essential micronutrients. Due to the complex nature of substrate selection by different transporters, increased expression and activity of transporters such as IRT1 result in excessive uptake of $\mathrm{Mn}, \mathrm{Ni}$, and $\mathrm{Zn}$, as well as some other heavy metals. Under such circumstances, vacuolar sequestration of excess heavy metals is extremely important (Eide et al., 1996; Korshunova et al., 1999; Baxter et al., 2008). Regulating substrate-specific vacuolar metal transporters, such as NRAMP3 and NRAMP4, can also eliminate toxic metals (such as Cd) from the food chain (Pottier et al., 2015). This could be achieved by developing plants that efficiently deposit toxic metals into root and/or shoot vacuoles, efficiently remobilizing the essential metals, while lacking the ability to remobilize toxic metals from the vacuole. HMA3 effectively performs this function in rice, contributing to significantly reduced toxic $\mathrm{Cd}$ in rice grains (Ueno et al., 2010; Miyadate et al., 2011; Sasaki et al., 2014). Changes in the regulation of genes involved in metal accumulation and/or homeostasis are also observed in plants exposed to different biotic and abiotic stressors (Expert et al., 2012; Lei et al., 2014; Rasheed et al., 2016), highlighting the importance of metal distribution to mitigate the harmful effects of these stressors. Thus, regulation of subcellular metal distribution/homeostasis may help plants adapt to diverse environmental conditions and exhibit tolerance to various biotic and abiotic stressors.

\section{CONCLUSION}

Regulation of cellular and subcellular metal homeostasis is extremely important for maintaining optimized metabolic rates and cellular functioning. The components of Fe homeostasis are understood reasonably well; however, the proteins involved in subcellular $\mathrm{Zn}$ and $\mathrm{Mn}$ and, to some extent, $\mathrm{Cu}$ homeostasis are poorly understood. Mutations in genes involved in metal uptake into the cell from the apoplasm often result in disturbed growth; however, mutations in genes involved in vacuolar metal homeostasis (e.g., VIT and NRAMPs) may positively affect growth and thus help in biofortification of crop plants. Fe, Zn, $\mathrm{Mn}$, and $\mathrm{Cu}$ are essential for a variety of metabolic reactions. Metabolome significantly changes under various abiotic stresses. Thus regulating the distribution of micronutrients to various subcellular compartments could be useful strategy to regulate metabolome under adverse climatic conditions. This could be achieved by using promoters, differentially regulating the expression of genes involved in subcellular metal transport under different abiotic stresses. In particular, maintaining the micronutrient balance between chloroplast, mitochondria and cytoplasm could significantly improve the metabolism and plant growth, particularly under stress conditions. Metabolic profiling of the mit-2 mutant demonstrated that subcellular metal distribution is important for regulating cellular metabolism, whereas osvit1 and osvit2 mutants clearly support the use of these proteins for biofortifying crop plants with essential micronutrients (Bashir et al., 2011b, 2013b; Zhang et al., 2012; Vigani et al., 2016). Although overexpressing AtVIT1 in 
cassava resulted in increased $\mathrm{Fe}$ accumulation demonstrating that AtVIT1 could significantly contribute to Fe biofortification (Narayanan et al., 2015). However, it should be noted that over accumulation of $\mathrm{Fe}$ in vacuole may leave the cytoplasm Fe deficient and ultimately disturbing the cell metabolism and plant growth. Thus, it would be reasonable to conclude that manipulating the mineral distribution in a particular subcellular compartment would significantly and specifically affect the cellular metabolism and accumulation of metals in leaves and seeds. Understanding the role of specific proteins in the distribution of metals inside cells provides opportunities to regulate their transport. This knowledge could be effectively used to optimize cellular metabolism leading to the development of crop plants with better nutritional value that are suitable for a diverse range of environments. Manipulating substrate specificity of transporters, such as NRAMP3 and NRAMP4, could

\section{REFERENCES}

Aguirre, G., and Pilon, M. (2016). Copper delivery to chloroplast proteins and its regulation. Front. Plant Sci. 6:1250. doi: 10.3389/fpls.2015.01250

Aoyama, T., Kobayashi, T., Takahashi, M., Nagasaka, S., Usuda, K., Kakei, Y., et al. (2009). OsYSL18 is a rice iron(III)-deoxymugineic acid transporter specifically expressed in reproductive organs and phloem of lamina joints. Plant Mol. Biol. 70, 681-692. doi: 10.1007/s11103-009-9500-3

Arrivault, S., Senger, T., and Krämer, U. (2006). The Arabidopsis metal tolerance protein AtMTP3 maintains metal homeostasis by mediating Zn exclusion from the shoot under Fe deficiency and Zn oversupply. Plant J. 46, 861-879. doi: 10.1111/j.1365-313X.2006.02746.x

Bashir, K., Hanada, K., Shimizu, M., Seki, M., Nakanishi, H., and Nishizawa, N. K. (2014). Transcriptomic analysis of rice in response to iron deficiency and excess. Rice 7, 18. doi: 10.1186/s12284-014-0018-1

Bashir, K., Ishimaru, Y., Itai, R., Senoura, T., Takahashi, M., An, G., et al. (2015). Iron deficiency regulated OsOPT7 is essential for iron homeostasis in rice. Plant Mol. Biol. 88, 165-176. doi: 10.1007/s11103-015-0315-0

Bashir, K., Ishimaru, Y., and Nishizawa, N. (2012). Molecular mechanisms of zinc uptake and translocation in rice. Plant Soil 361, 189-201. doi: 10.1007/s11104012-1240-5

Bashir, K., Ishimaru, Y., and Nishizawa, N. K. (2011a). Identification and characterization of the major mitochondrial Fe transporter in rice. Plant Signal. Behav. 6, 1591-1593. doi: 10.4161/psb.6.10.17132

Bashir, K., Ishimaru, Y., Shimo, H., Nagasaka, S., Fujimoto, M., Takanashi, H., et al. (2011b). The rice mitochondrial iron transporter is essential for plant growth. Nat. Commun. 2:322. doi: 10.1038/ncomms1326

Bashir, K., Nagasaka, S., Itai, R., Kobayashi, T., Takahashi, M., Nakanishi, H., et al. (2007). Expression and enzyme activity of glutathione reductase is upregulated by Fe-deficiency in graminaceous plants. Plant Mol. Biol. 65, 277-284.

Bashir, K., Nozoye, T., Ishimaru, Y., Nakanishi, H., and Nishizawa, N. K. (2013a). Exploiting new tools for iron bio-fortification of rice. Biotechnol. Adv. 31, 1624-1633. doi: 10.1016/j.biotechadv.2013.08.012

Bashir, K., Takahashi, R., Akhtar, S., Ishimaru, Y., Nakanishi, H., and Nishizawa, N. K. (2013b). The knockdown of OsVIT2 and MIT affects iron localization in rice seed. Rice 6, 1-7. doi: 10.1186/1939-8433-6-31

Baxter, I. R., Vitek, O., Lahner, B., Muthukumar, B., Borghi, M., Morrissey, J., et al. (2008). The leaf ionome as a multivariable system to detect a plant's physiological status. Proc. Natl. Acad. Sci. U.S.A. 105, 12081-12086. doi: 10.1073/pnas.0804175105

Bernard, D. G., Cheng, Y., Zhao, Y., and Balk, J. (2009). An allelic mutant series of ATM3 reveals its key role in the biogenesis of cytosolic iron-sulfur proteins in Arabidopsis. Plant Physiol. 151, 590-602. doi: 10.1104/pp.109. 143651

Binder, B. M., Rodríguez, F. I., and Bleecker, A. B. (2010). The copper transporter RAN1 is essential for biogenesis of ethylene receptors in Arabidopsis. J. Biol. Chem. 285, 37263-37270. doi: 10.1074/jbc.M110.170027 also help breed crop plants that accumulate more beneficial minerals while reducing toxic metal contents in the edible parts.

\section{AUTHOR CONTRIBUTIONS}

$\mathrm{KB}$ wrote the article. KB, SR, TK, MS, and NN discussed and revised the manuscript.

\section{ACKNOWLEDGMENTS}

KB is supported by RIKEN (Japan) through the RIKEN foreign postdoctoral fellowship (FPR) and SR is supported by the RIKEN JRA program.

Boutigny, S., Sautron, E., Finazzi, G., Rivasseau, C., Frelet-Barrand, A., Pilon, M., et al. (2014). HMA1 and PAA1, two chloroplast-envelope PIB-ATPases, play distinct roles in chloroplast copper homeostasis. J. Exp. Bot. 65, 1529-1540. doi: 10.1093/jxb/eru020

Briat, J.-F., Duc, C., Ravet, K., and Gaymard, F. (2010). Ferritins and iron storage in plants. Biochim. Biophys. Acta (BBA) 1800, 806-814. doi: 10.1016/j.bbagen.2009.12.003

Broadley, M. R., White, P. J., Hammond, J. P., Zelko, I., and Lux, A. (2007). Zinc in plants. New Phytol. 173, 677-702. doi: 10.1111/j.1469-8137.2007.01996.x

Catty, P., Boutigny, S., Miras, R., Joyard, J., Rolland, N., and Seigneurin-Berny, D. (2011). Biochemical characterization of AtHMA6/PAA1, a chloroplast envelope Cu (I)-ATPase. J. Biol. Chem. 286, 36188-36197. doi: 10.1074/jbc.M111.241034

Chen, Z., Fujii, Y., Yamaji, N., Masuda, S., Takemoto, Y., Kamiya, T., et al. (2013). Mn tolerance in rice is mediated by MTP8.1, a member of the cation diffusion facilitator family. J. Exp. Bot. 64, 4375-4387. doi: 10.1093/jxb/ert243

Conte, S. S., Chu, H. H., Chan-Rodriguez, D., Punshon, T., Vasques, K. A., Salt, D. E., et al. (2013). Arabidopsis thaliana Yellow Stripe1-Like4 and Yellow Stripe1-Like6 localize to internal cellular membranes and are involved in metal ion homeostasis. Front. Plant Sci. 4:283. doi: 10.3389/fpls.2013.00283

Conte, S. S., and Walker, E. L. (2011). Transporters contributing to iron trafficking in plants. Mol. Plant 4, 464-476. doi: 10.1093/mp/ssr015

Delhaize, E., Gruber, B. D., Pittman, J. K., White, R. G., Leung, H., Miao, Y., et al. (2007). A role for the AtMTP11 gene of Arabidopsis in manganese transport and tolerance. Plant J. 51, 198-210. doi: 10.1111/j.1365-313X.2007.03138.x

Desbrosses-Fonrouge, A.-G., Voigt, K., Schröder, A., Arrivault, S., Thomine, S., and Krämer, U. (2005). Arabidopsis thaliana MTP1 is a Zn transporter in the vacuolar membrane which mediates $\mathrm{Zn}$ detoxification and drives leaf $\mathrm{Zn}$ accumulation. FEBS Lett. 579, 4165-4174. doi: 10.1016/j.febslet.2005.06.046

Divol, F., Couch, D., Conéjéro, G., Roschzttardtz, H., Mari, S., and Curie, C. (2013). The Arabidopsis yellow stripe like 4 and 6 transporters control iron release from the chloroplast. Plant Cell 25, 1040-1055. doi: 10.1105/tpc.112.107672

Duy, D., Stübe, R., Wanner, G., and Philippar, K. (2011). The chloroplast permease PIC1 regulates plant growth and development by directing homeostasis and transport of iron. Plant Physiol. 155, 1709-1722. doi: 10.1104/pp.110.170233

Eide, D., Broderius, M., Fett, J., and Guerinot, M. L. (1996). A novel iron-regulated metal transporter from plants identified by functional expression in yeast. Proc. Natl. Acad. Sci. U.S.A. 93, 5624-5628. doi: 10.1073/pnas.93.11.5624

Eroglu, S., Meier, B., von Wirén, N., and Peiter, E. (2016). The vacuolar manganese transporter MTP8 determines tolerance to iron deficiency-induced chlorosis in Arabidopsis. Plant Physiol. 170, 1030-1045. doi: 10.1104/pp.15.01194

Expert, D., Franza, T., and Dellagi, A. (2012). "Iron in plant-pathogen interactions," in Molecular Aspects of Iron Metabolism in Pathogenic and Symbiotic Plant-Microbe Associations, eds D. Expert, O’Brian, and R. Mark (Heidelberg: Springer), 7-39.

Finazzi, G., Petroutsos, D., Tomizioli, M., Flori, S., Sautron, E., Villanova, V., et al. (2015). Ions channels/transporters and chloroplast regulation. Cell Calcium 58, 86-97. doi: 10.1016/j.ceca.2014.10.002 
Garcia-Molina, A., Andrés-Colás, N., Perea-García, A., del Valle-Tascón, S., Peñarrubia, L., and Puig, S. (2011). The intracellular Arabidopsis COPT5 transport protein is required for photosynthetic electron transport under severe copper deficiency. Plant J. 65, 848-860. doi: 10.1111/j.1365-313X.2010.04472.x

Gollhofer, J., Timofeev, R., Lan, P., Schmidt, W., and Buckhout, T. J. (2014). Vacuolar-iron-transporter1-like proteins mediate iron homeostasis in Arabidopsis. PLoS ONE 9:e110468. doi: 10.1371/journal.pone.0110468

Grillet, L., Ouerdane, L., Flis, P., Hoang, M. T. T., Isaure, M.-P., Lobinski, R., et al. (2014). Ascorbate efflux as a new strategy for iron reduction and transport in plants. J. Biol. Chem. 289, 2515-2525. doi: 10.1074/jbc.M113.514828

Grotz, N., and Guerinot, M. L. (2006). Molecular aspects of $\mathrm{Cu}, \mathrm{Fe}$ and $\mathrm{Zn}$ homeostasis in plants. Biochim. Biophys. Acta Mol. Cell Res. 1763, 595-608. doi: 10.1016/j.bbamcr.2006.05.014

Haydon, M. J., and Cobbett, C. S. (2007). A novel major facilitator superfamily protein at the tonoplast influences zinc tolerance and accumulation in Arabidopsis. Plant Physiol. 143, 1705-1719. doi: 10.1104/pp.106.092015

Haydon, M. J., Kawachi, M., Wirtz, M., Hillmer, S., Hell, R., and Krämer, U. (2012). Vacuolar nicotianamine has critical and distinct roles under iron deficiency and for zinc sequestration in Arabidopsis. Plant Cell 24, 724-737. doi: 10.1105/tpc.111.095042

Ishimaru, Y., Bashir, K., Fujimoto, M., An, G., Itai, R. N., Tsutsumi, N., et al. (2009). Rice-specific mitochondrial iron-regulated gene (MIR) plays an important role in iron homeostasis. Mol. Plant 2, 1059-1066. doi: 10.1093/mp/ssp051

Ishimaru, Y., Bashir, K., Nakanishi, H., and Nishizawa, N. K. (2012). OsNRAMP5, a major player for constitutive iron and manganese uptake in rice. Plant Signal. Behav. 7, 763-766. doi: 10.4161/psb.20510

Ishimaru, Y., Bashir, K., and Nishizawa, N. K. (2011). Zn uptake and translocation in rice plants. Rice 4, 21-27. doi: 10.1007/s12284-011-9061-3

Jain, A. (2014). Ferric reductases and transporters that contribute to mitochondrial iron homeostasis, Ph.D. thesis, University of South Carolina, Columbia, WA.

Jain, A., Wilson, G. T., and Connolly, E. L. (2014). The diverse roles of FRO family metalloreductases in iron and copper homeostasis. Front. Plant Sci. 5:100. doi: 10.3389/fpls. 2014.00100

Jeong, J., Cohu, C., Kerkeb, L., Pilon, M., Connolly, E. L., and Guerinot, M. L. (2008). Chloroplast Fe(III) chelate reductase activity is essential for seedling viability under iron limiting conditions. Proc. Natl. Acad. Sci. U.S.A. 105, 10619-10624. doi: 10.1073/pnas.0708367105

Jeong, J., and Guerinot, M. L. (2009). Homing in on iron homeostasis in plants. Trends Plant Sci. 14, 280-285. doi: 10.1016/j.tplants.2009.02.006

Kawachi, M., Kobae, Y., Mori, H., Tomioka, R., Lee, Y., and Maeshima, M. (2009). A mutant strain Arabidopsis thaliana that lacks vacuolar membrane zinc transporter MTP1 revealed the latent tolerance to excessive zinc. Plant Cell Physiol. 50, 1156-1170. doi: 10.1093/pcp/pcp067

Kim, S. A., Punshon, T., Lanzirotti, A., Li, L., Alonso, J. M., Ecker, J. R., et al. (2006). Localization of iron in Arabidopsis seed requires the vacuolar membrane transporter VIT1. Science 314, 1295-1298. doi: 10.1126/science.1132563

Kim, Y.-Y., Choi, H., Segami, S., Cho, H.-T., Martinoia, E., Maeshima, M., et al. (2009). AtHMA1 contributes to the detoxification of excess $\mathrm{Zn}$ (II) in Arabidopsis. Plant J. 58, 737-753. doi: 10.1111/j.1365-313X.2009.03818.x

Klaumann, S., Nickolaus, S. D., Fürst, S. H., Starck, S., Schneider, S., Ekkehard Neuhaus, H., et al. (2011). The tonoplast copper transporter COPT5 acts as an exporter and is required for interorgan allocation of copper in Arabidopsis thaliana. New Phytol. 192, 393-404. doi: 10.1111/j.1469-8137.2011.03798.x

Kobae, Y., Uemura, T., Sato, M. H., Ohnishi, M., Mimura, T., Nakagawa, T., et al. (2004). Zinc transporter of Arabidopsis thaliana AtMTP1 is localized to vacuolar membranes and implicated in zinc homeostasis. Plant Cell Physiol. 45, 1749-1758. doi: 10.1093/pcp/pci015

Kobayashi, T., and Nishizawa, N. K. (2012). Iron Uptake, translocation, and regulation in higher plants. Annu. Rev. Plant Biol. 63, 131-152. doi: 10.1146/annurev-arplant-042811-105522

Kobayashi, T., and Nishizawa, N. K. (2014). Iron sensors and signals in response to iron deficiency. Plant Sci. 224, 36-43. doi: 10.1016/j.plantsci.2014.04.002

Korshunova, Y. O., Eide, D., Gregg Clark, W., Lou Guerinot, M., and Pakrasi, H. B. (1999). The IRT1 protein from Arabidopsis thaliana is a metal transporter with a broad substrate range. Plant Mol. Biol. 40, 37-44. doi: 10.1023/a:10264386 15520

Kushnir, S., Babiychuk, E., Storozhenko, S., Davey, M. W., Papenbrock, J., De Rycke, R., et al. (2001). A mutation of the mitochondrial ABC transporter Stal leads to dwarfism and chlorosis in the Arabidopsis mutant starik. Plant Cell 13, 89-100. doi: 10.1105/tpc.13.1.89

Lanquar, V., Lelievre, F., Bolte, S., Hames, C., Alcon, C., Neumann, D., et al. (2005). Mobilization of vacuolar iron by AtNRAMP3 and AtNRAMP4 is essential for seed germination on low iron. EMBO J. 24, 4041-4051. doi: 10.1038/sj.emboj.7600864

Lasswell, J., Rogg, L. E., Nelson, D. C., Rongey, C., and Bartel, B. (2000). Cloning and characterization of IAR1, a gene required for auxin conjugate sensitivity in Arabidopsis. Plant Cell 12, 2395-2408. doi: 10.1105/tpc.12.12.2395

Lei, G. J., Zhu, X. F., Wang, Z. W., Dong, F., Dong, N. Y., and Zheng, S. J. (2014). Abscisic acid alleviates iron deficiency by promoting root iron reutilization and transport from root to shoot in Arabidopsis. Plant Cell Environ. 37, 852-863. doi: $10.1111 /$ pce. 12203

Lill, R., Dutkiewicz, R., Freibert, S. A., Heidenreich, T., Mascarenhas, J., Netz, D. J., et al. (2015). The role of mitochondria and the CIA machinery in the maturation of cytosolic and nuclear iron-sulfur proteins. Eur. J. Cell Biol. 94, 280-291. doi: 10.1016/j.ejcb.2015.05.002

López-Millán, A. F., Duy, D., and Philippar, K. (2016). Chloroplast iron transport proteins - function and impact on plant physiology. Front. Plant Sci. 7:178. doi: 10.3389/fpls.2016.00178

Marschner, H. (1995). Mineral Nutrition of Higher Plants. London: Academic Press.

Mary, V., Schnell Ramos, M., Gillet, C., Socha, A., Giraudat, J., Agorio, A., et al. (2015). Bypassing iron storage in endodermal vacuoles rescues the iron mobilization defect in the nramp3nramp4 double mutant. Plant Physiol. 169, 748-759. doi: 10.1104/pp.15.00380

Mei, H., Cheng, N. H., Zhao, J., Park, S., Escareno, R. A., Pittman, J. K., et al. (2009). Root development under metal stress in Arabidopsis thaliana requires the H+/cation antiporter CAX4. New Phytol. 183, 95-105. doi: 10.1111/j.14698137.2009.02831.x

Millar, A. H., Whelan, J., Soole, K. L., and Day, D. A. (2011). Organization and regulation of mitochondrial respiration in plants. Annu. Rev. Plant Biol. 62, 79-104.

Milner, M. J., Seamon, J., Craft, E., and Kochian, L. V. (2013). Transport properties of members of the ZIP family in plants and their role in $\mathrm{Zn}$ and Mn homeostasis. J. Exp. Bot. 64, 369-381. doi: 10.1093/jxb/ers315

Miyadate, H., Adachi, S., Hiraizumi, A., Tezuka, K., Nakazawa, N., Kawamoto, T., et al. (2011). OsHMA3, a P1B-type of ATPase affects root-to-shoot cadmium translocation in rice by mediating efflux into vacuoles. New Phytol. 189, 190199. doi: 10.1111/j.1469-8137.2010.03459.x

Momonoi, K., Yoshida, K., Mano, S., Takahashi, H., Nakamori, C., Shoji, K., et al. (2009). A vacuolar iron transporter in tulip, TgVit1, is responsible for blue coloration in petal cells through iron accumulation. Plant J. 59, 437-447. doi: 10.1111/j.1365-313X.2009.03879.x

Morel, M., Crouzet, J., Gravot, A., Auroy, P., Leonhardt, N., Vavasseur, A., et al. (2009). AtHMA3, a P1B-ATPase allowing $\mathrm{Cd} / \mathrm{Zn} / \mathrm{Co} / \mathrm{Pb}$ vacuolar storage in Arabidopsis. Plant Physiol. 149, 894-904. doi: 10.1104/pp.108. 130294

Morrissey, J., Baxter, I. R., Lee, J., Li, L., Lahner, B., Grotz, N., et al. (2009). The Ferroportin metal efflux proteins function in iron and cobalt homeostasis in Arabidopsis. Plant Cell 21, 3326-3338. doi: 10.1105/tpc.109.069401

Murgia, I., and Vigani, G. (2015). Analysis of Arabidopsis thaliana atfer4-1, atfh and atfer4-1/atfh mutants uncovers frataxin and ferritin contributions to leaf ionome homeostasis. Plant Physiol. Biochem. 94, 65-72. doi: 10.1016/j.plaphy.2015.05.011

Narayanan, N., Beyene, G., Chauhan, R. D., Gaitán-Solis, E., Grusak, M. A., Taylor, N., et al. (2015). Overexpression of Arabidopsis VIT1 increases accumulation of iron in cassava roots and stems. Plant Sci. 240, 170-181. doi: 10.1016/j.plantsci.2015.09.007

Nouet, C., Motte, P., and Hanikenne, M. (2011). Chloroplastic and mitochondrial metal homeostasis. Trends Plant Sci. 16, 395-404. doi: 10.1016/j.tplants.2011.03.005

Nozoye, T., Nagasaka, S., Bashir, K., Takahashi, M., Kobayashi, T., Nakanishi, H., et al. (2014a). Nicotianamine synthase 2 localizes to the vesicles of irondeficient rice roots, and its mutation in the $\operatorname{YXX} \varphi$ or LL motif causes the disruption of vesicle formation or movement in rice. Plant J. 77, 246-260. doi: $10.1111 /$ tpj.12383

Nozoye, T., Nagasaka, S., Kobayashi, T., Takahashi, M., Sato, Y., Sato, Y., et al. (2011). Phytosiderophore efflux transporters are crucial for iron 
acquisition in graminaceous plants. J. Biol. Chem. 286, 5446-5454. doi: 10.1074/jbc.M110.180026

Nozoye, T., Tsunoda, K., Nagasaka, S., Bashir, K., Takahashi, M., Kobayashi, T., et al. (2014b). Rice nicotianamine synthase localizes to particular vesicles for proper function. Plant Signal. Behav. 9:e28660. doi: 10.4161/psb.28660

Palmer, C. M., and Guerinot, M. L. (2009). Facing the challenges of Cu, Fe and $\mathrm{Zn}$ homeostasis in plants. Nat. Chem. Biol. 5, 333-340. doi: 10.1038/nchembio.166

Pedas, P., Stokholm, M. S., Hegelund, J. N., Ladegård, A. H., Schjoerring, J. K., and Husted, S. (2014). Golgi localized barley MTP8 proteins facilitate Mn transport. PLoS ONE 9:e113759. doi: 10.1371/journal.pone.0113759

Peng, J.-S., and Gong, J.-M. (2014). Vacuolar sequestration capacity and long-distance metal transport in plants. Front. Plant Sci. 5:19. doi: 10.3389/fpls.2014.00019

Pottier, M., Oomen, R., Picco, C., Giraudat, J., Scholz-Starke, J., Richaud, P., et al. (2015). Identification of mutations allowing natural resistance associated macrophage proteins (NRAMP) to discriminate against cadmium. Plant J. 83, 625-637. doi: 10.1111/tpj.12914

Rasheed, S., Bashir, K., Matsui, A., Tanaka, M., and Seki, M. (2016). Transcriptomic analysis of soil-grown Arabidopsis thaliana roots and shoots in response to a drought stress. Front. Plant Sci. 7:180. doi: 10.3389/fpls.2016.00180

Remy, E., Cabrito, T. R., Batista, R. A., Hussein, M. A., Teixeira, M. C., Athanasiadis, A., et al. (2014). Intron retention in the $5^{\prime}$ UTR of the novel ZIF2 transporter enhances translation to promote zinc tolerance in Arabidopsis. PLoS Genet. 10:e1004375. doi: 10.1371/journal.pgen.1004375

Remy, E., Cabrito, T. R., Batista, R. A., Teixeira, M. C., Sá-Correia, I., and Duque, P. (2015). The major facilitator superfamily transporter ZIFL2 modulates cesium and potassium homeostasis in Arabidopsis. Plant Cell Physiol. 56, 148-162. doi: $10.1093 / \mathrm{pcp} / \mathrm{pcu} 157$

Roschzttardtz, H., Grillet, L., Isaure, M.-P., Conéjéro, G., Ortega, R., Curie, C., et al. (2011). Plant cell nucleolus as a hot spot for iron. J. Biol. Chem. 286, 27863-27866. doi: 10.1074/jbc.C111.269720

Sasaki, A., Yamaji, N., and Ma, J. F. (2014). Overexpression of OsHMA3 enhances $\mathrm{Cd}$ tolerance and expression of $\mathrm{Zn}$ transporter genes in rice. J. Exp. Bot. 65, 6013-6021. doi: 10.1093/jxb/eru340

Sasaki, A., Yamaji, N., Xia, J., and Ma, J. F. (2011). OsYSL6 is involved in the detoxification of excess manganese in rice. Plant Physiol. 157, 1832-1840. doi: 10.1104/pp.111.186031

Schaaf, G., Honsbein, A., Meda, A. R., Kirchner, S., Wipf, D., and von Wirén, N. (2006). AtIREG2 encodes a tonoplast transport protein involved in irondependent nickel detoxification in Arabidopsis thaliana roots. J. Biol. Chem. 281, 25532-25540. doi: 10.1074/jbc.M601062200

Schaedler, T. A., Thornton, J. D., Kruse, I., Schwarzländer, M., Meyer, A. J., van Veen, H. W., et al. (2014). A conserved mitochondrial ATP-binding cassette transporter exports glutathione polysulfide for cytosolic metal cofactor assembly. J. Biol. Chem. 289, 23264-23274. doi: 10.1074/jbc.M114. 553438

Schmidt, W., and Buckhout, T. J. (2011). A hitchhiker's guide to the Arabidopsis ferrome. Plant Physiol. Biochem. 49, 462-470. doi: 10.1016/j.plaphy.2010.12.001

Schwarzländer, M., König, A.-C., Sweetlove, L. J., and Finkemeier, I. (2012). The impact of impaired mitochondrial function on retrograde signalling: a meta-analysis of transcriptomic responses. J. Exp. Bot. 63, 1735-1750. doi: 10.1093/jxb/err374

Seo, P. J., Park, J., Park, M.-J., Kim, Y.-S., Kim, S.-G., Jung, J.-H., et al. (2012). A Golgi-localized MATE transporter mediates iron homoeostasis under osmotic stress in Arabidopsis. Biochem. J. 442, 551-561. doi: 10.1042/BJ20111311

Shanmugam, V., Wang, Y.-W., Tsednee, M., Karunakaran, K., and Yeh, K.-C. (2015). Glutathione plays an essential role in nitric oxide-mediated irondeficiency signaling and iron-deficiency tolerance in Arabidopsis. Plant J. 84, 464-477. doi: 10.1111/tpj.13011
Socha, A. L., and Guerinot, M. L. (2014). Mn-euvering manganese: the role of transporter gene family members in manganese uptake and mobilization in plants. Front. Plant Sci. 5:106. doi: 10.3389/fpls.2014.00106

Suzuki, M., Bashir, K., Inoue, H., Takahashi, M., Nakanishi, H., and Nishizawa, N. (2012). Accumulation of starch in Zn-deficient rice. Rice 5:9. doi: 10.1186/19398433-5-9

Sweetlove, L. J., Fait, A., Nunes-Nesi, A., Williams, T., and Fernie, A. R. (2007). The Mitochondrion: an integration point of cellular metabolism and signalling. Critic. Rev. Plant Sci. 26, 17-43. doi: 10.1080/07352680601147919

Tarantino, D., Morandini, P., Ramirez, L., Soave, C., and Murgia, I. (2011). Identification of an Arabidopsis mitoferrin like carrier protein involved in Fe metabolism. Plant Physiol. Biochem. 49, 520-529. doi: 10.1016/j.plaphy.2011.02.003

Teschner, J., Lachmann, N., Schulze, J., Geisler, M., Selbach, K., SantamariaAraujo, J., et al. (2010). A novel role for Arabidopsis mitochondrial ABC transporter ATM3 in molybdenum cofactor biosynthesis. Plant Cell 22, 468480. doi: 10.1105/tpc.109.068478

Ueno, D., Yamaji, N., Kono, I., Huang, C. F., Ando, T., Yano, M., et al. (2010). Gene limiting cadmium accumulation in rice. Proc. Natl. Acad. Sci. U.S.A. 107, 16500-16505. doi: 10.1073/pnas.1005396107

Vest, K. E., Wang, J., Gammon, M. G., Maynard, M. K., White, O. L., Cobine, J. A., et al. (2016). Overlap of copper and iron uptake systems in mitochondria in Saccharomyces cerevisiae. Open Biol. 6, 150223. doi: 10.1098/rsob.150223

Vigani, G., Bashir, K., Ishimaru, Y., Lehmann, M., Casiraghi, M., Nakanishi, H., et al. (2016). Knocking down mitochondrial iron transporter (MIT) reprograms primary and secondary metabolism in rice plants. J. Exp. Bot. 67, 1357-1368. doi: $10.1093 /$ jxb/erv531

Vigani, G., Zocchi, G., Bashir, K., Philippar, K., and Briat, J.-F. (2013a). Cellular iron homeostasis and metabolism in plant. Front. Plant Sci. 4:490. doi: 10.3389/fpls.2013.00490

Vigani, G., Zocchi, G., Bashir, K., Philippar, K., and Briat, J.-F. (2013b). Signals from chloroplasts and mitochondria for iron homeostasis regulation. Trends Plant Sci. 18, 305-311. doi: 10.1016/j.tplants.2013.01.006

Williams, L. E., and Mills, R. F. (2005). P1B-ATPases - an ancient family of transition metal pumps with diverse functions in plants. Trends Plant Sci. 10, 491-502. doi: 10.1016/j.tplants.2005.08.008

Yoshida, K., and Negishi, T. (2013). The identification of a vacuolar iron transporter involved in the blue coloration of cornflower petals. Phytochemistry 94, 60-67. doi: 10.1016/j.phytochem.2013.04.017

Yruela, I. (2009). Copper in plants: acquisition, transport and interactions. Funct. Plant Biol. 36, 409-430. doi: 10.1071/FP08288

Zhang, Y., Xu, Y.-H., Yi, H.-Y., and Gong, J.-M. (2012). Vacuolar membrane transporters OsVIT1 and OsVIT2 modulate iron translocation between flag leaves and seeds in rice. Plant J. 72, 400-410. doi: 10.1111/j.1365313X.2012.05088.x

Zheng, L., Fujii, M., Yamaji, N., Sasaki, A., Yamane, M., Sakurai, I., et al. (2011). Isolation and characterization of a barley yellow stripe-like gene, HvYSL5. Plant Cell Physiol. 52, 765-774. doi: 10.1093/pcp/pcr009

Conflict of Interest Statement: The authors declare that the research was conducted in the absence of any commercial or financial relationships that could be construed as a potential conflict of interest.

Copyright (c) 2016 Bashir, Rasheed, Kobayashi, Seki and Nishizawa. This is an openaccess article distributed under the terms of the Creative Commons Attribution License (CC BY). The use, distribution or reproduction in other forums is permitted, provided the original author(s) or licensor are credited and that the original publication in this journal is cited, in accordance with accepted academic practice. No use, distribution or reproduction is permitted which does not comply with these terms. 